\title{
Thermodynamic formulation of flowing soft matter with transient forces
}

\section{Journal Article}

Author(s):

Savin, Thierry; Briels, Wim J.; Öttinger, Hans Christian (1)

Publication date:

2013-01

Permanent link:

https://doi.org/10.3929/ethz-b-000063844

Rights / license:

In Copyright - Non-Commercial Use Permitted

Originally published in:

Rheologica Acta 52(1), https://doi.org/10.1007/s00397-012-0661-7 


\title{
Thermodynamic formulation of flowing soft matter with transient forces
}

\author{
Thierry Savin • Wim J. Briels • \\ Hans Christian Öttinger
}

Received: 18 June 2012 / Revised: 24 October 2012 / Accepted: 30 October 2012 / Published online: 28 November 2012

(C) Springer-Verlag Berlin Heidelberg 2012

\begin{abstract}
The Responsive Particle Dynamics model is a very efficient method to account for the transient forces present in complex fluids, such as solutions of entangled polymers. This coarse-grained model considers a solution of particles that are made of a core and a corona. The cores typically interact through conservative interactions, while the coronae transiently penetrate each other to form short-lived temporary interactions, typically of entropic origin. In this study, we reformulate the resulting rheological model within the general framework of nonequilibrium thermodynamics called General Equation for the Nonequilibrium ReversibleIrreversible Coupling. This allows us to determine the consistency of the model, from a mechanistic and thermodynamic point of view, and to isolate the reversible and irreversible contributions to the dynamics of the model system.
\end{abstract}

Keywords Soft matter $\cdot$ RaPiD • Nonequilibrium • GENERIC

\section{Introduction}

The theoretical description of flowing complex fluids ultimately involves a certain level of coarse graining

T. Savin $(\varangle) \cdot$ H. C. Öttinger

Department of Materials, ETH Zürich, 8093 Zürich, Switzerland

e-mail: savin@mat.ethz.ch

W. J. Briels

Computational Biophysics, University of Twente,

P.O. Box 217, 7500 AE Enschede, The Netherlands
(Larson 1998; Cates and Evans 2000; Müller-Plathe 2002; Kröger 2005; Ilg et al. 2009). Indeed, not all degrees of freedom can be accounted for in full details. In fact, a full description would be inconsequential because most experiments and observations essentially probe only a subset of all these degrees of freedom, called "slow variables" (Öttinger 2005; Briels 2009). Polymer liquids are the archetypical example of complex fluids, and the internal degrees of freedom, such as the positions of all molecules, as well as their orientations and their configurations, are not accessible to typical bulk rheology measurements (Bird et al. 1987). The outcomes of the measurements may strongly depend, however, on some of the microscopic details, like for example the chains' entanglement, whose manifest effects include the occurrence of shear thinning and shear banding of the fluid under deformation (Dhont and Briels 2008; Boukany and Wang 2009; Briels et al. 2011; Adams et al. 2011; Cao and Likhtman 2012). An intricate challenge of developing a theory for flowing polymer solutions is to isolate a small number of intelligible coarse-grained, slow variables, whose transport equations can be expressed in term of a small number of material properties.

A few years ago, in part motivated by developing efficient algorithms for soft matter simulations, Briels and coworkers introduced a particulate nonequilibrium model of flowing complex fluids that includes transient forces between the particles (van den Noort et al. 2007; Kindt and Briels 2007). Their universal model, the Responsive Particle Dynamics model (RaPiD), considers a collection of particles, whose interactions depend on a small number of variables describing the history of the eliminated degrees of freedom. To be more precise, the particles interact through conservative forces 


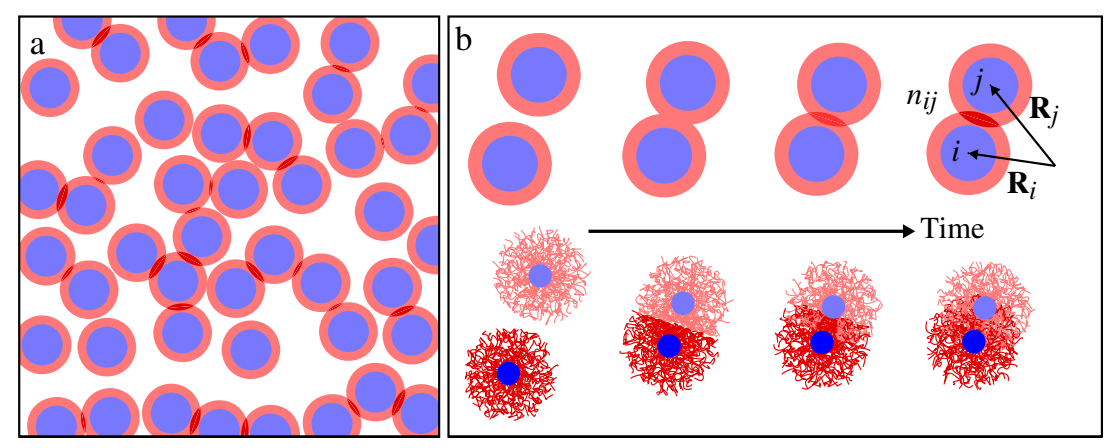

Fig. 1 We consider here a suspension of interacting RaPiD particles (a), each being composed of a core and a corona. The coronae interact via transient forces, which are relaxing at time scales comparable to the dynamics of the particles' center of mass, and the pair penetration order parameter $n_{i j}$, between particles $i$ and $j$ quantifies the interaction between the coronae (b). The RaPiD model can represent a variety of systems (see text for examples and corresponding references), and we show in $\mathbf{b}$ the case of star polymers: $n_{i j}$ could represent the number of entanglements in that case representing cores of various degrees of softness surrounded by possibly very complex coronae (see Fig. 1) and, in addition to these, through transient forces, depending on the actual thermodynamic state of the coronae. Central to their model is the definition of a dynamic pair penetration order parameter $n_{i j}$ between particles $i$ and $j$ (Fig. 1b) that quantifies the deviation from equilibrium of the overlapping coronae of both particles. The penetration parameter could, for example, indicate the degree of mixing between the arms of two star polymers (see Fig. 1b) or the number of bridges established between telechelic micelles. The dynamics of the coronae's penetration is assumed to be comparable to the dynamics of the particles' centers of mass, put in motion by moving boundaries, pressure gradients, and/or by Brownian fluctuations. The RaPiD model has been successful in reporting rheological behavior of star polymers (Briels et al. 2011), telechelic (Sprakel et al. 2009), sticky particles and composites (Padding et al. 2011). Notably, RaPiD is one of the rare techniques for efficiently incorporating memory into Brownian dynamics simulations, although the modecoupling theory was used to also obtain usable timedependent correlation functions for the description of glasses (Götze and Sjörgen 1992; Götze 1999; Fuchs and Cates 2009).

An important issue that has not yet been addressed with the RaPiD model is its "thermodynamic admissibility". It is essential that the final transport equations satisfy the principles of thermodynamics, and one needs to identify the thermodynamic potentials that generate them. The General Equation for the Nonequilibrium Reversible-Irreversible Coupling (GENERIC) framework provides a convenient and rigorous tool to assess the mechanical and thermodynamic validity of transport equations while highlighting the separation of reversible and irreversible dynamics contributions (Öttinger and Grmela 1997; Grmela and Öttinger 1997; Öttinger 2005). It has been successful in both proving the consistency, as well as extending a wide variety of models, including rheological models of colloids (Wagner 2001; Ellero et al. 2003) and polymer solutions (Öttinger 2001), elasticity (Mielke 2011) and viscoplasticity (Hütter and Svendsen 2012), and even relativistic hydrodynamics (Öttinger 1998a, b, 1999; Ilg and Öttinger 1999) or dissipative quantum systems (Öttinger 2011).

In this article, we propose to use GENERIC to build a complete thermodynamic model of transient interaction in complex fluids, which ultimately verifies the validity of the RaPiD model. In a first part, we briefly summarize the GENERIC paradigm, that we next apply to the RaPiD model, before discussing several implications of our new formulation.

\section{The GENERIC framework}

Transport equations for nonequilibrium systems have a well-defined structure in which reversible and irreversible contributions can be specified separately. The reversible contribution is "under mechanistic control", and hence of the Hamiltonian form, requiring an underlying geometric structure based on Poisson brackets. The remaining irreversible contribution is generated by the nonequilibrium entropy by means of a dissipative bracket.

In this article, we will base our discussion on the GENERIC formulation of time evolution for nonequilibrium systems. If $A$ is an arbitrary observable, that is, a sufficiently regular real-valued function or functional of a set of variables $\mathbf{x}$ required for a complete 
description of a given nonequilibrium system, the time evolution of $A$ is given by

$\frac{d A}{d t}=\{A, E\}+[A, S]$.

The observables $E$ and $S$ generating time evolution are the total energy and entropy, and $\{\cdot, \cdot\}$ and $[\cdot, \cdot]$ are Poisson and dissipative brackets, respectively. The bracket of two observables $A$ and $B$ is another observable with a linear dependence on $A$ and $B$ (a more complete characterization of Poisson and dissipative brackets is given below). The two contributions to the time evolution of $A$ generated by the total energy $E$ and the entropy $S$ in Eq. 1 are the reversible and irreversible contributions, respectively. Equation 1 is supplemented by the complementary degeneracy requirements

$\{S, A\}=0$,

and

$[E, A]=0$,

which hold for all observables $A$. The requirement that the entropy is a degenerate functional of the Poisson bracket expresses the reversible nature of the first contribution to the dynamics: the functional form of the entropy is such that it cannot be affected by the Poisson bracket contribution to the dynamics, no matter which observable $A$ is used as a generator of reversible dynamics. The requirement that the energy is a degenerate functional of the dissipative bracket expresses the conservation of the total energy by the dissipative contribution to the dynamics in a closed system.

For completeness, we give the defining properties of Poisson and dissipative brackets. The Poisson bracket possesses the antisymmetry property

$\{A, B\}=-\{B, A\}$,

and satisfies the product, or Leibniz, rule

$\{A B, C\}=A\{B, C\}+B\{A, C\}$,

as well as the Jacobi identity

$\{A,\{B, C\}\}+\{B,\{C, A\}\}+\{C,\{A, B\}\}=0$,

where $A, B$, and $C$ are arbitrary observables. These properties are well-known from the Poisson brackets of classical mechanics, and they express the essence of reversible dynamics. The Jacobi identity (6), which is a highly restrictive condition for formulating proper reversible dynamics, expresses the invariance of Poisson brackets in the course of time (time-structure invariance).

The dissipative bracket satisfies the symmetry condition $^{1}$

$[A, B]=[B, A]$,

and the non-negativeness condition

$[A, A] \geq 0$.

This non-negativeness condition, together with the degeneracy requirement (2), guarantees that the entropy is a nondecreasing function of time,

$\frac{d S}{d t}=[S, S] \geq 0$.

The condition (8) may hence be regarded as a strong formulation of the second law of nonequilibrium thermodynamics.

In practical calculations, it is often convenient to formulate GENERIC in terms of Poisson and friction operators instead of brackets. More precisely, one can write, without loss of generality,

$\{A, B\}=\frac{\delta A}{\delta \mathbf{x}} \cdot \mathbf{L}(\mathbf{x}) \cdot \frac{\delta B}{\delta \mathbf{x}}$,

and

$[A, B]=\frac{\delta A}{\delta \mathbf{x}} \cdot \mathbf{M}(\mathbf{x}) \cdot \frac{\delta B}{\delta \mathbf{x}}$,

where $\mathbf{L}(\mathbf{x})$ is the Poisson operator, $\mathbf{M}(\mathbf{x})$ is the friction operator, $\mathbf{x}$ is the vector of system variables, and $\frac{\delta A}{\delta \mathbf{x}}$ designates the functional derivatives of $A$ with respect to $\mathbf{x}$. The Leibniz rule (5) then follows automatically, $\mathbf{L}$ is imposed to be antisymmetric by Eq. 4, and $\mathbf{M}$ must be symmetric and positive semi-definite from Eqs. 7-8. The time-evolution equations for the system variables $\mathbf{x}$ implied by Eq. 1 can be expressed in the form

$\frac{d \mathbf{x}}{d t}=\mathbf{L}(\mathbf{x}) \cdot \frac{\delta E}{\delta \mathbf{x}}+\mathbf{M}(\mathbf{x}) \cdot \frac{\delta S}{\delta \mathbf{x}}$,

\footnotetext{
${ }^{1}$ For a more detailed discussion of the Onsager-Casimir symmetry of the dissipative bracket, see Section 3.2.1 in Öttinger (2005); for a very detailed level of description, the dissipative bracket might not possess any well-defined symmetry properties, as is elaborated in Section 7.2.4 of Öttinger (2005).
} 
and the degeneracy conditions, Eqs. 2 and 3, respectively follow:

$$
\begin{aligned}
\mathbf{L}(\mathbf{x}) \cdot \frac{\delta S}{\delta \mathbf{x}} & =0, \\
\mathbf{M}(\mathbf{x}) \cdot \frac{\delta E}{\delta \mathbf{x}} & =0 .
\end{aligned}
$$

The summations implied by the dot product include integrations over the system's volume when fields are involved in the list of independent variables.

We now implement the GENERIC formulation of the RaPiD model by choosing proper variables $\mathbf{x}$ and by building each block $E(\mathbf{x}), S(\mathbf{x}), \mathbf{L}(\mathbf{x})$, and $\mathbf{M}(\mathbf{x})$ of the evolution equation (12).

\section{Coarse-grained model}

\section{System variables $\mathbf{x}$}

The general coarse-grained model to describe the dynamics for a system of particles with transient interactions has been introduced by van den Noort et al. (2007) and Briels (2009). The model is both applicable to solutions of complex molecules as well as to melts of such molecules. In the former application, the solvent is considered to be a continuum, assumed here to be Newtonian, while in all cases, the particles are treated explicitly. The particles interact via conservative forces and transient forces depending on a set of pair penetration order parameters treated as dynamical variables. The time evolution of the particles and the pair penetration order parameters is assumed to be governed by Brownian displacements. In the GENERIC formulation, they are conveniently represented by their probability distribution. We may therefore introduce the following complete set of independent slow variables:

- $\rho(\mathbf{r})$ is the total mass density of the system,

- $\mathbf{m}(\mathbf{r})$ the total momentum density of the system,

- $\epsilon(\mathbf{r})$ the background internal energy density,

- $\psi(\mathbf{R}, \mathbf{n})$ the probability distribution of the state $(\mathbf{R}, \mathbf{n})$.

Here, we have denoted $\mathbf{r}$ the position in the system's volume, $\mathbf{R}=\left\{\mathbf{R}_{i}\right\}_{1 \leq i \leq N}$ the $N$ particles' positions (i.e., $\mathcal{N}=3 N$ coordinates), and $\mathbf{n}=\left\{n_{i j}\right\}_{1 \leq i, j \leq N}$ the pair penetration order parameters (i.e., $\mathcal{P}=N(N-1) / 2$ distinct values). The latter can be interpreted as "the number of stickers" or the "number of entanglements" between the coronae of two particles, although we consider in general that it can take any real value. The pair penetration parameters follow a relaxation dynamics whose time scale is comparable to the dynamics of the particles' positions (Briels et al. 2011).

Note that $\rho$ and $\mathbf{m}$ measure the densities for the total system composed of the RaPiD particles, usually complex polymers, together with the solvent in which they bath, while we separate the energetic and entropic contributions of the particles and of the solvent: here, $\epsilon$ is the internal energy density of the solvent only. The internal energy generated by the particles' interactions is explicitly given in the next section. We further assume that the RaPiD particles are neutrally buoyant in the solvent so that any gravitational effects can be ignored. If the modeled material does not have a solvent, such as a melt, then $\epsilon$ is the background internal energy of the remaining fast fluctuations in the system.

The vector of independent variables is written

$\mathbf{x}(\mathbf{r}, \mathbf{R}, \mathbf{n})=[\rho(\mathbf{r}), \mathbf{m}(\mathbf{r}), \epsilon(\mathbf{r}), \psi(\mathbf{R}, \mathbf{n})]^{\mathrm{T}}$

and depends on the position $\mathbf{r}$ in the system, the particles positions $\mathbf{R}$, and the order parameters $\mathbf{n}$. The time dependency will not be explicitly indicated throughout the article.

Total energy $E(\mathbf{x})$ and entropy $S(\mathbf{x})$

The expressions for the total energy $E(\mathbf{x})$ and the total entropy $S(\mathbf{x})$ are as follows:

$$
\begin{aligned}
E(\mathbf{x})= & \int\left[\frac{\mathbf{m}(\mathbf{r})^{2}}{2 \rho(\mathbf{r})}+\epsilon(\mathbf{r})\right] d^{3} r \\
& +\iint \Phi^{E}(\mathbf{R}) \psi(\mathbf{R}, \mathbf{n}) d^{\mathcal{P}} n d^{\mathcal{N}} R, \\
S(\mathbf{x})= & \int s(\rho(\mathbf{r}), \epsilon(\mathbf{r})) d^{3} r \\
& -k_{\mathrm{B}} \iint \psi(\mathbf{R}, \mathbf{n}) \ln \frac{\psi(\mathbf{R}, \mathbf{n})}{\psi_{0}} d^{\mathcal{P}} n d^{\mathcal{N} R} \\
& +\iint \Phi^{S}(\mathbf{R}, \mathbf{n}) \psi(\mathbf{R}, \mathbf{n}) d^{\mathcal{P}} n d^{\mathcal{N}} R,
\end{aligned}
$$

where $\Phi^{E}(\mathbf{R})$ is the energy resulting from the conservative interactions between the RaPiD particles, depending on the positions $\mathbf{R}$ only, $s(\rho, \epsilon)$ is the entropy density of the solvent, and $\Phi^{S}(\mathbf{R}, \mathbf{n})$ is the configurational entropy associated with the coronae in the system's state $(\mathbf{R}, \mathbf{n})$.

The first term of $E(\mathbf{x})$ in Eq. 16, with only a spatial integration over $\mathbf{r}$, represents the total kinetic energy of the system and the background internal energy. The second term, involving a configurational integration 
over ( $\mathbf{R}, \mathbf{n})$, gives the additional conservative contribution of the potential interaction between the RaPiD particles. In most cases, we may write

$$
\Phi^{E}(\mathbf{R})=\sum_{i<j} \phi^{E}\left(\mathbf{R}_{i j}\right),
$$

where $\mathbf{R}_{i j}=\mathbf{R}_{i}-\mathbf{R}_{j}$ is the position of the $j$ th particle, relative to the position of particle $i$, and the sum runs over all pairs of indiscernible particles.

We now explain the various entropic contributions present in the expression (17) for $S(\mathbf{x})$. The first term is the background entropy of the system, written as the spatial integral of the background entropy density $s(\rho, \epsilon)$, which is a well-defined thermodynamic quantity by virtue of the local equilibrium assumption, depending only on the mass density $\rho$ and the internal energy density $\epsilon$. The existence of the local "background" entropy and energy, $s$ and $\epsilon$ respectively, that are independent of the configurational coordinates $(\mathbf{R}, \mathbf{n})$ notably ensures that a background temperature and chemical potential can be defined (see later). The second term of $S(\mathbf{x})$ is a configurational entropy contribution that represents the preference of the system towards a uniform distribution, and $\psi_{0}$ is a characteristic scale of $\psi$ whose choice has no consequence. The third term introduces the total configurational entropy $\Phi^{S}(\mathbf{R}, \mathbf{n})$ of all particles' coronae for the given configuration $(\mathbf{R}, \mathbf{n})$. We separate the equilibrium configurational entropy of the overlapping coronae from the nonequilibrium entropic contributions caused by insufficient relaxation on mutual penetration of the coronae, and we may write

$\Phi^{S}(\mathbf{R}, \mathbf{n})=\Sigma^{S}(\mathbf{R})+\Psi^{S}(\mathbf{R}, \mathbf{n})$.

We assume the following form for $\Sigma^{S}(\mathbf{R})$,

$\Sigma^{S}(\mathbf{R})=\sum_{i} \sigma\left(\rho_{i}(\mathbf{R})\right)$

as the sum of configurational entropies of the $N$ coronae, where $\sigma$ is the entropy of the corona of particle $i$ that depends only on the local density $\rho_{i}$ of the surrounding constituents for the RaPiD particle $i$. For a particular set of positions for the RaPiD particles, the local microscopic density is well defined at every position in the system. The entropy of the coronae may then be uniquely calculated by distributing the material that makes up the coronae, for example by a Flory-Huggins type of calculation in the case of polymeric coronae. The entropy per RaPiD particle will then depend on the local microscopic density. The latter local density $\rho_{i}$ depends only on the relative locations $\mathbf{R}_{i j}$ of the $N-1$ particles surrounding the particle $i$, and may, apart from some constant factor, be written as

$\rho_{i}(\mathbf{R})=\sum_{j} w\left(\mathbf{R}_{i j}\right)$

where $w$ is a weight function whose integral over the entire space equals unity, and where $i$, hence the reference density $w(\mathbf{0})$ contributed by an isolated particle, must be included in the sum. Details about this choice for $\Sigma^{S}$ can be found in the articles by Pagonabarraga and Frenkel (2001), Kindt and Briels (2007), and Briels (2009).

The penetration term $\Psi^{S}(\mathbf{R}, \mathbf{n})$ in the definition (Eq. 19) involves the sum over all pairs of particles of the entropy due to insufficient relaxation of interpenetrated coronae:

$\Psi^{S}(\mathbf{R}, \mathbf{n})=-\sum_{i<j} \sum^{S}\left(\mathbf{R}_{i j}, n_{i j}\right)$

Here, $-\phi^{S}\left(\mathbf{R}_{i j}, n_{i j}\right)$ is the stored, nonequilibrium, instantaneous entropy in the penetrating coronae of the pair $i, j$ of two RaPiD particles, providing a negative contribution because it is removed from the total entropy of the system (hence the minus sign for $\phi^{S} \geq 0$, see below). An expression for the entropy loss $\phi^{S}\left(\mathbf{R}_{i j}, n_{i j}\right)$ has been proposed as the leading term in an expansion with respect to $n_{i j}-n_{0}\left(\mathbf{R}_{i j}\right)$, where $n_{0}\left(\mathbf{R}_{i j}\right)$ is the equilibrium value of the penetration order parameter (van den Noort et al. 2007; Briels 2009; Briels et al. 2011). We thus write:

$\phi^{S}\left(\mathbf{R}_{i j}, n_{i j}\right)=\frac{1}{2} \alpha^{S}\left(\mathbf{R}_{i j}\right)\left[n_{i j}-n_{0}\left(\mathbf{R}_{i j}\right)\right]^{2}+\ldots$,

where the stiffness $\alpha^{S}\left(\mathbf{R}_{i j}\right)=\frac{\partial^{2} \phi^{S}}{\partial n_{i j}^{2}}>0$ is a design parameter that may indeed depend on the interparticle distance. Various forms of $n_{0}\left(\mathbf{R}_{i j}\right)$, depending on what molecule the RaPiD particle is representing (star polymer, core-shell particle, ... ), may be found (Kindt and Briels 2007; Padding et al. 2011; Briels et al. 2011). Note however that the general model presented here does not depend on this particular choice for $\phi^{S}\left(\mathbf{R}_{i j}, n_{i j}\right)$. Its validity, however, is linked to the specific dependency of $\Phi^{S}$ on $\mathbf{R}_{i j}$ highlighted in the formulas (20) to (22).

Equipped with the formulas (16) and (17) for the total energy $E(\mathbf{x})$ and the total entropy $S(\mathbf{x})$ of the nonequilibrium system, we calculate their functional 
derivatives with respect to each component of the vector of variables $\mathbf{x}$ given by the definition (15):

$$
\begin{aligned}
& \frac{\delta E}{\delta \mathbf{x}}=\left[-\frac{\mathbf{v}(\mathbf{r})^{2}}{2}, \mathbf{v}(\mathbf{r}), 1, \Phi^{E}(\mathbf{R})\right]^{\mathrm{T}}, \\
& \frac{\delta S}{\delta \mathbf{x}}=\left[-\frac{\mu(\mathbf{r})}{T(\mathbf{r})}, 0, \frac{1}{T(\mathbf{r})}, \frac{\delta S}{\delta \psi}\right]^{\mathrm{T}},
\end{aligned}
$$

with the velocity defined as $\mathbf{v}(\mathbf{r})=\frac{\mathbf{m}(\mathbf{r})}{\rho(\mathbf{r})}$, the temperature as $T(\mathbf{r})=\left(\left.\frac{\partial s(\rho, \epsilon)}{\partial \epsilon}\right|_{\rho}\right)^{-1}$, and the chemical potential as $\mu(\mathbf{r})=-\left.T(\mathbf{r}) \frac{\partial S(\rho, \epsilon)}{\partial \rho}\right|_{\epsilon}$. The last component $\frac{\delta S}{\delta \psi}$ reads:

$$
\frac{\delta S}{\delta \psi}=\Phi^{S}(\mathbf{R}, \mathbf{n})-k_{\mathrm{B}} \ln \frac{\psi(\mathbf{R}, \mathbf{n})}{\psi_{0}},
$$

to within an arbitrary constant, irrelevant in the present study, coming from calculating the functional derivative with the normalization constrain $\int \psi(\mathbf{R}$, n) $d^{\mathcal{P}} n d^{\mathcal{N}} R=1$. There remains now to express the operators $\mathbf{L}(\mathbf{x})$ and $\mathbf{M}(\mathbf{x})$ to complete the GENERIC formulation.

\section{Operators $\mathbf{L}$ and $\mathbf{M}$}

The procedure to implement the matrices $\mathbf{L}$ and $\mathbf{M}$ has been detailed in the original GENERIC articles (Öttinger and Grmela 1997; Grmela and Öttinger 1997). The Poisson operator $\mathbf{L}$ reads:

$$
\begin{aligned}
\mathbf{L}(\mathbf{x})= & -\left[\begin{array}{cccr}
0 & \frac{\partial}{\partial \mathbf{r}} \rho & 0 & 0 \\
\rho \frac{\partial}{\partial \mathbf{r}}\left(\frac{\partial}{\partial \mathbf{r}} \mathbf{m}+\mathbf{m} \frac{\partial}{\partial \mathbf{r}}\right)^{\mathrm{T}} \epsilon \frac{\partial}{\partial \mathbf{r}}+\frac{\partial}{\partial \mathbf{r}} p & 0 \\
0 & \frac{\partial}{\partial \mathbf{r}} \epsilon+p \frac{\partial}{\partial \mathbf{r}} & 0 & 0 \\
0 & 0 & 0 & 0
\end{array}\right] \\
& -\left[\begin{array}{cccc}
0 & 0 & 0 & 0 \\
0 & 0 & \frac{\partial}{\partial \mathbf{r}} \cdot \boldsymbol{\Pi}^{S} & \sum_{k} \psi \delta\left(\mathbf{r}-\mathbf{R}_{k}\right) \frac{\partial}{\partial \mathbf{R}_{k}} \\
0 & \boldsymbol{\Pi}^{S} \cdot \frac{\partial}{\partial \mathbf{r}} & 0 & 0 \\
0 \sum_{k} \frac{\partial}{\partial \mathbf{R}_{k}} \delta\left(\mathbf{r}-\mathbf{R}_{k}\right) \psi & 0 & 0
\end{array}\right] .
\end{aligned}
$$

In order to simplify the notation, we have performed one spatial integration implied by the dot product when $\mathbf{L}(\mathbf{x})$ operates on $\frac{\delta E}{\delta \mathbf{x}}$, even when this apparently breaks the manifest antisymmetry of the operator. As a consequence, all differential operators act on everything to the right, i.e., including $\frac{\delta E}{\delta \mathbf{x}}$. Such simplification, valid for a local theory, is merely conventional (Öttinger 2005, Section 2.2.2). Also, the dependencies on $\mathbf{r}, \mathbf{R}$, and $\mathbf{n}$ are not all displayed in the components of $\mathbf{L}$, and we refer the reader to the previous section for the corresponding dependencies of the various functions $\rho, \mathbf{m}, \Phi^{E}, \Phi^{S}$, $\psi, \ldots$ etc.

The first matrix in the above expression of $\mathbf{L}$ corresponds to the reversible dynamic involving terms in $\rho, \mathbf{m}$, and $\epsilon$ and has been calculated in previous works (Öttinger and Grmela 1997; Öttinger 2005). In the components $L_{\epsilon \mathbf{m}}$ and $L_{\mathbf{m} \epsilon}$ of this matrix, we have introduced the isotropic, hydrostatic pressure $p=T s-\epsilon+$ $\mu \rho$. The second matrix of Eq. 27 is associated with the RaPiD particles. In this matrix, the additional component $L_{\psi \mathbf{m}}=\sum_{k} \frac{\partial}{\partial \mathbf{R}_{k}} \delta\left(\mathbf{r}-\mathbf{R}_{k}\right) \psi$ convects the probability distribution under the motion of the particles, while the component $L_{\epsilon \mathbf{m}}$ allows for an extra pressure tensor, $\Pi^{S}(\mathbf{r})$. The component $L_{\mathbf{m} \psi}$ and $L_{\mathbf{m} \epsilon}$ are obtained from
$L_{\psi \mathbf{m}}$ and $L_{\epsilon \mathbf{m}}$, respectively, using the antisymmetry requirement, Eq. 4, of the Poisson operator.

The expression of $\Pi^{S}(\mathbf{r})$ is obtained, to within a divergence-free contribution, to satisfy the degeneracy requirement. The condition $\mathbf{L} \cdot \frac{\delta S}{\delta \mathbf{x}}=0$ is immediately met, except for the second row of $\mathbf{L}$, and the $\mathbf{m}$ component of the vector $\mathbf{L} \cdot \frac{\delta S}{\delta \mathbf{x}}$ reads

$$
\begin{aligned}
\left(\mathbf{L} \cdot \frac{\delta S}{\delta \mathbf{x}}\right)_{\mathbf{m}}= & \rho \frac{\partial}{\partial \mathbf{r}} \frac{\mu}{T}-\epsilon \frac{\partial}{\partial \mathbf{r}} \frac{1}{T}-\frac{\partial}{\partial \mathbf{r}} \frac{p}{T}-\frac{\partial}{\partial \mathbf{r}} \cdot \frac{\Pi^{S}}{T} \\
& -\iint\left[\sum_{k} \delta\left(\mathbf{r}-\mathbf{R}_{k}\right) \frac{\partial \Phi^{S}}{\partial \mathbf{R}_{k}}\right] \psi d^{\mathcal{P}} n d^{\mathcal{N} R} \\
& +k_{\mathrm{B}} \iint\left[\sum_{k} \delta\left(\mathbf{r}-\mathbf{R}_{k}\right) \frac{\partial \psi}{\partial \mathbf{R}_{k}}\right] d^{\mathcal{P}} n d^{\mathcal{N}} R .
\end{aligned}
$$

By virtue of the local equilibrium, the first three terms of the above expression can be written:

$$
\frac{\partial}{\partial \mathbf{r}}\left(\rho \frac{\mu}{T}-\epsilon \frac{1}{T}-\frac{p}{T}+s\right)=0
$$


To obtain degeneracy and the form of the extra pressure tensor, we now verify that the integral terms of Eq. 28 can be written in a divergence form. The first integral involves the entropy $\Phi^{S}$ of the coronae, that we separate into two contributions using Eq. 19. For the first term $\Sigma^{S}(\mathbf{R})$, we use the relations (20) and (21) to calculate $\frac{\partial \Sigma^{S}}{\partial \mathbf{R}_{k}}=\sum_{i} \frac{d \sigma\left(\rho_{i}\right)}{d \rho_{i}} \frac{d \rho_{i}(\mathbf{R})}{d \mathbf{R}_{k}}$, with $\frac{d \rho_{i}(\mathbf{R})}{d \mathbf{R}_{k}}=\frac{d w\left(\mathbf{R}_{i k}\right)}{d \mathbf{R}_{k}}=-\frac{d w\left(\mathbf{R}_{i k}\right)}{d \mathbf{R}_{i k}}$ for $i \neq k$ and $\frac{d \rho_{k}(\mathbf{R})}{d \mathbf{R}_{k}}=\sum_{j} \frac{d w\left(\mathbf{R}_{k j}\right)}{d \mathbf{R}_{k}}=\sum_{j \neq k} \frac{d w\left(\mathbf{R}_{k j}\right)}{d \mathbf{R}_{k j}}$ (the term $w(\mathbf{0})$ disappears after differentiation with respect to $\left.\mathbf{R}_{k}\right)$. We thus verify $\frac{\partial \Sigma^{S}}{\partial \mathbf{R}_{k}}=\sum_{i \neq k}\left[\frac{d \sigma\left(\rho_{k}\right)}{d \rho_{k}} \frac{d w\left(\mathbf{R}_{k i}\right)}{d \mathbf{R}_{k i}}-\right.$ $\left.\frac{d \sigma\left(\rho_{i}\right)}{d \rho_{i}} \frac{d w\left(\mathbf{R}_{i k}\right)}{d \mathbf{R}_{i k}}\right]$, from which we obtain $\sum_{k} \delta\left(\mathbf{r}-\mathbf{R}_{k}\right) \frac{\partial \Sigma^{s}}{\partial \mathbf{R}_{k}}=$ $\sum \sum_{i \neq j}\left[\delta\left(\mathbf{r}-\mathbf{R}_{i}\right)-\delta\left(\mathbf{r}-\mathbf{R}_{j}\right)\right] \frac{d \sigma}{d \rho_{i}} \frac{d w}{d \mathbf{R}_{i i}}$. This well-known transformation, first used by Irving and Kirkwood (1950), introduces the difference of two delta functions, that can next be expressed as (McLennan 1989)

$$
\begin{aligned}
\delta\left(\mathbf{r}-\mathbf{R}_{i}\right)-\delta\left(\mathbf{r}-\mathbf{R}_{j}\right) & =\int_{0}^{1} \frac{\partial}{\partial \lambda} \delta\left(\mathbf{r}-\mathbf{R}_{j}-\lambda \mathbf{R}_{i j}\right) d \lambda \\
& =-\frac{\partial}{\partial \mathbf{r}} \cdot\left(\Delta_{i j} \mathbf{R}_{i j}\right),
\end{aligned}
$$

where

$\Delta_{i j}(\mathbf{r})=\int_{0}^{1} \delta\left(\mathbf{r}-\mathbf{R}_{j}-\lambda \mathbf{R}_{i j}\right) d \lambda$

is a generalized spatial delta-function that signals the segment connecting the RaPiD particles $i$ and $j$, and has the dimension of an inverse volume. Finally, we can write

$\sum_{k} \delta\left(\mathbf{r}-\mathbf{R}_{k}\right) \frac{\partial \Sigma^{S}}{\partial \mathbf{R}_{k}}=-\frac{\partial}{\partial \mathbf{r}} \cdot\left(\sum_{i \neq j} \sum_{i j} \Delta_{i j} \frac{d \sigma}{d \rho_{i}} \frac{d w}{d \mathbf{R}_{i j}}\right)$

in a divergence form. Following a similar reasoning, we use the expansion of $\Psi^{S}$ given by Eq. 22. Since $\sum_{k} \delta\left(\mathbf{r}-\mathbf{R}_{k}\right) \frac{\partial}{\partial \mathbf{R}_{k}} \phi^{S}\left(\mathbf{R}_{i j}, n_{i j}\right)=\left[\delta\left(\mathbf{r}-\mathbf{R}_{i}\right)-\delta(\mathbf{r}-\right.$ $\left.\left.\mathbf{R}_{j}\right)\right] \frac{\partial}{\partial \mathbf{R}_{i}} \phi^{S}\left(\mathbf{R}_{i j}, n_{i j}\right)$, we can again use Eq. 30 to finally find a divergence form of

$\sum_{k} \delta\left(\mathbf{r}-\mathbf{R}_{k}\right) \frac{\partial \Psi^{S}}{\partial \mathbf{R}_{k}}=\frac{\partial}{\partial \mathbf{r}} \cdot\left(\sum \sum_{i<j} \Delta_{i j} \mathbf{R}_{i j} \frac{\partial \phi^{S}}{\partial \mathbf{R}_{i j}}\right)$.

In the last integral term of Eq. 28, one can interchange the derivative in $\mathbf{R}_{k}$ by a derivative with respect to $\mathbf{r}$ to find its divergence form

$k_{\mathrm{B}} \iint\left[\sum_{k} \delta\left(\mathbf{r}-\mathbf{R}_{k}\right) \frac{\partial \psi}{\partial \mathbf{R}_{k}}\right] d^{\mathcal{P}} n d^{\mathcal{N}} R=\frac{\partial}{\partial \mathbf{r}} \cdot\left(c k_{\mathrm{B}} \mathbf{1}\right)$, with $c(\mathbf{r})=\iint\left[\sum_{k} \delta\left(\mathbf{r}-\mathbf{R}_{k}\right)\right] \psi d^{\mathcal{P}} n d^{\mathcal{N}} R$ the concentration of RaPiD particles in the solution, that has the dimension of an inverse volume. We finally find the expression of $\boldsymbol{\Pi}^{S}$ for which $\left(\mathbf{L} \cdot \frac{\delta S}{\delta \mathbf{x}}\right)_{\mathbf{m}}$ vanishes:

$$
\begin{aligned}
\Pi^{S}=\iint T & {\left[\left(\sum_{i \neq j} \sum_{i j} \mathbf{R}_{i j} \frac{d \sigma}{d \rho_{i}} \frac{d w}{d \mathbf{R}_{i j}}\right)\right.} \\
& \left.-\left(\sum \sum_{i<j} \Delta_{i j} \mathbf{R}_{i j} \frac{\partial \phi^{S}}{\partial \mathbf{R}_{i j}}\right)\right] \psi d^{\mathcal{P}} n d^{\mathcal{N} R} \\
& +c k_{\mathrm{B}} T \mathbf{1} .
\end{aligned}
$$

All terms in $\Pi^{S}$ have an entropic origin, the integral term coming from the coronae, while the last term is the classical osmotic pressure for the ideal colloidal solution.

Verification of the Jacobi identity for the above operator $\mathbf{L}$ can be done using symbolic computational software (Kröger and Hütter 2010), using an action of the group of space deformations on the configurational variables, or by hand.

The friction operator $\mathbf{M}$ gives the dissipative contributions to the transport equations. As such, it requires the introduction of transport coefficients, that are essential material properties of the system. Similar to the Poisson matrix, we may identify two contributions of the M matrix to the irreversible dynamics. The first contribution is coming from the solvent's rheological response (this part vanishes in the absence of solvent) and from the system's thermal behavior. We assume that the solvent is a Newtonian isotropic fluid, with viscosity $\eta$ and dilatational viscosity $\kappa$, and that the heat flux $\mathbf{j}$ in the system follows Fourier's law of conduction, $\mathbf{j}(\mathbf{r})=-\lambda(\mathbf{r}) \cdot \frac{\partial T}{\partial \mathbf{r}}$. The tensorial nature of the thermal conductivity $\lambda$ allows for anisotropic heat transport, and its r-dependency may be inherited from inhomogeneous fields in the system.

The second contribution of the $\mathbf{M}$ operator to the transport equations contains the relaxation of the particles' positional and structural configurations, and involves some diffusivity tensors. The diffusivity tensor D indicates the hydrodynamic interaction of the RaPiD particles with the fluid background. We ignore hydrodynamic interaction between the particles, and we may eventually write $\mathbf{D}=D_{0} \mathbf{1}$ with $D_{0}$ the particles' size-dependent Stokes-Einstein diffusion coefficient. We also introduce a generalized, scalar coefficient of diffusion $B$ for the propagation of the penetration parameter. 
We can finally write, separating the two contributions in the order we discussed them:

$$
\begin{aligned}
& \mathbf{M}(\mathbf{x})=\left[\begin{array}{cccr}
0 & 0 & 0 & 0 \\
0-\left(\frac{\partial}{\partial \mathbf{r}} \eta T \frac{\partial}{\partial \mathbf{r}}+\mathbf{1} \frac{\partial}{\partial \mathbf{r}} \cdot \eta T \frac{\partial}{\partial \mathbf{r}}\right)^{\mathrm{T}}-\frac{\partial}{\partial \mathbf{r}} \hat{\kappa} T \frac{\partial}{\partial \mathbf{r}} & \frac{\partial}{\partial \mathbf{r}} \cdot \eta T \dot{\boldsymbol{\gamma}}+\frac{\partial}{\partial \mathbf{r}} \frac{\hat{\kappa} T}{2} \operatorname{tr} \dot{\boldsymbol{\gamma}} & 0 \\
0 & -\eta T \dot{\boldsymbol{\gamma}} \cdot \frac{\partial}{\partial \mathbf{r}}-\frac{\hat{\kappa} T}{2} \operatorname{tr} \dot{\boldsymbol{\gamma}} \frac{\partial}{\partial \mathbf{r}} & \frac{\eta T}{2} \dot{\boldsymbol{\gamma}}: \dot{\boldsymbol{\gamma}}+\frac{\hat{\kappa} T}{4}(\operatorname{tr} \dot{\boldsymbol{\gamma}})^{2}-\frac{\partial}{\partial \mathbf{r}} \cdot \lambda \cdot T^{2} \frac{\partial}{\partial \mathbf{r}} & 0 \\
0 & 0 & 0 & 0
\end{array}\right]
\end{aligned}
$$

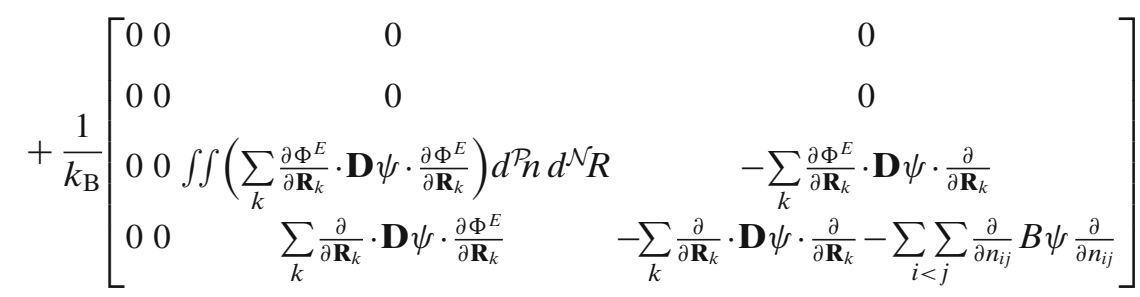

where $\hat{\kappa}=\kappa-\frac{2}{3} \eta$ is the translated dilatational viscosity, $\dot{\gamma}(\mathbf{r})=\frac{\partial}{\partial \mathbf{r}} \mathbf{v}(\mathbf{r})+\left[\frac{\partial}{\partial \mathbf{r}} \mathbf{v}(\mathbf{r})\right]^{\mathrm{T}}$ is the symmetrized velocity gradient tensor, and tri $\dot{\gamma}$ its trace.

The formula for the hydrodynamic part, that is the first matrix in Eq. 36, has been derived in the original GENERIC paper (Öttinger and Grmela 1997) where its degeneracy condition is also verified. The second part of $\mathbf{M}$ is built in the following way: first, $M_{\psi \psi}$ is introduced to account for the diffusive dissipation of the RaPiD particles and of their pair penetration parameter, then $M_{\psi \epsilon}$ is obtained by using the degeneracy condition, while the symmetry of $\mathbf{M}$ gives $M_{\epsilon \psi}$; we finally get $M_{\epsilon \epsilon}$ by applying the degeneracy criterion once again. The condition $\mathbf{M} \cdot \frac{\delta E}{\delta \mathbf{x}}=0$ for the above matrix is thus obtained by construction. In the above

expression of $\mathbf{M}$, we have neglected any hydrodynamic interactions between the RaPiD particles. Note finally that the spatial integration has also been performed here in order to simplify the notation.

Time-evolution equations

Using the GENERIC Eq. (12), the time-evolution equations for the total mass density, the total momentum density, the solvent internal energy density and the state probability distribution are, respectively:

$$
\begin{aligned}
\frac{\partial \rho}{\partial t} & =-\frac{\partial}{\partial \mathbf{r}} \cdot(\mathbf{v} \rho), \\
\frac{\partial \mathbf{m}}{\partial t} & =-\frac{\partial}{\partial \mathbf{r}} \cdot(\mathbf{v m})-\frac{\partial}{\partial \mathbf{r}} \cdot \boldsymbol{\pi},
\end{aligned}
$$

$\frac{\partial \epsilon}{\partial t}=-\frac{\partial}{\partial \mathbf{r}} \cdot(\mathbf{v} \epsilon+\mathbf{j})-\left(p \mathbf{1}+\boldsymbol{\tau}+\boldsymbol{\Pi}^{S}\right):\left(\frac{\partial}{\partial \mathbf{r}} \mathbf{v}\right)+\iint \sum_{k} \frac{\partial \Phi^{E}}{\partial \mathbf{R}_{k}} \cdot \mathbf{D} \cdot\left[\frac{\partial \psi}{\partial \mathbf{R}_{k}}+\psi \frac{\partial}{\partial \mathbf{R}_{k}}\left(\frac{\Phi}{k_{\mathrm{B}} T}\right)\right] d^{\mathcal{P}} n d^{\mathcal{N}} R$,

$\frac{\partial \psi}{\partial t}=-\sum_{k} \frac{\partial}{\partial \mathbf{R}_{k}} \cdot\left[\mathbf{v}\left(\mathbf{R}_{k}\right) \psi\right]+\sum_{k} \frac{\partial}{\partial \mathbf{R}_{k}} \cdot \mathbf{D} \cdot\left[\frac{\partial \psi}{\partial \mathbf{R}_{k}}+\psi \frac{\partial}{\partial \mathbf{R}_{k}}\left(\frac{\Phi}{k_{\mathrm{B}} T}\right)\right]+\sum_{i<j} \sum_{i j} \frac{\partial}{\partial n_{i j}} B\left[\frac{\partial \psi}{\partial n_{i j}}+\psi \frac{\partial}{\partial n_{i j}}\left(\frac{\Phi}{k_{\mathrm{B}} T}\right)\right]$.

In the momentum balance (38), we have defined the total pressure tensor

$\pi=p \mathbf{1}+\boldsymbol{\tau}+\Pi$,

where the total osmotic pressure tensor, $\Pi=\Pi^{E}+\Pi^{S}$ includes the energetic contribution

$\boldsymbol{\Pi}^{E}=-\iint\left(\sum_{i<j} \sum_{i j} \mathbf{R}_{i j} \frac{\partial \phi^{E}}{\partial \mathbf{R}_{i j}}\right) \psi d^{\mathcal{P}} n d^{\mathcal{N}} R$.
We expressed the latter using the following equality

$$
\sum_{k} \delta\left(\mathbf{r}-\mathbf{R}_{k}\right) \frac{\partial \Phi^{E}}{\partial \mathbf{R}_{k}}=-\frac{\partial}{\partial \mathbf{r}} \cdot\left(\sum_{i<j} \sum_{i j} \mathbf{R}_{i j} \frac{\partial \phi^{E}}{\partial \mathbf{R}_{i j}}\right),
$$

derived by following the same reasoning outlined in the previous section to introduce the difference of deltafunctions, Eq. 30. We also included in Eq. 41 the viscous stress tensor $\boldsymbol{\tau}$, that takes Newton's form, $\tau=-\eta \dot{\gamma}-$ $\hat{\kappa} \frac{\partial}{\partial \mathbf{r}} \cdot \mathbf{v 1}$. 
In the energy and probability transport Eqs. (39) and (40), we introduced the free energy $\Phi(\mathbf{r}, \mathbf{R}, \mathbf{n})=$ $\Phi^{E}(\mathbf{R})-T(\mathbf{r}) \Phi^{S}(\mathbf{R}, \mathbf{n})$.

From the Smoluchowski Eq. (40), we can infer the following stochastic evolution equations for the RaPiD particles' positions:

$\frac{d \mathbf{R}_{k}}{d t}=\mathbf{v}\left(\mathbf{R}_{k}\right)-\mathbf{D} \cdot \frac{\partial}{\partial \mathbf{R}_{k}}\left(\frac{\Phi}{k_{\mathrm{B}} T}\right)+\sqrt{2 \mathbf{D}} \cdot \boldsymbol{\Theta}_{k}$,

and for the penetration parameters:

$\frac{d n_{i j}}{d t}=-B \frac{\partial}{\partial n_{i j}}\left(\frac{\Phi}{k_{\mathrm{B}} T}\right)+\sqrt{2 B} \theta_{i j}$,

where $\boldsymbol{\Theta}_{k}$ and $\theta_{i j}$ are respectively a vector and a scalar realization of delta correlated, independent stationary Gaussian random processes with zero-mean that verify

$\left\langle\Theta_{k, v}(t) \Theta_{k^{\prime}, v^{\prime}}\left(t^{\prime}\right)\right\rangle=\delta_{k k^{\prime}} \delta_{v v^{\prime}} \delta\left(t-t^{\prime}\right)$,

$\left\langle\theta_{i j}(t) \theta_{i^{\prime} j^{\prime}}\left(t^{\prime}\right)\right\rangle=\delta_{i i^{\prime}} \delta_{j j^{\prime}} \delta\left(t-t^{\prime}\right)$,

at all times $t$ and $t^{\prime}$, where $\delta_{i j}$ is the Kronecker delta and $\Theta_{k, v}$ indicates the $v$-component of the vector $\boldsymbol{\Theta}_{k}$.

With the choice of $\phi^{S}\left(\mathbf{R}_{i j}, n_{i j}\right)$ given by Eq. 23 , the time-evolution equation of the penetration parameter reads

$\frac{d n_{i j}}{d t}=-\frac{n_{i j}-n_{0}}{\tau}+\sqrt{2 B} \theta_{i j}$,

where $\tau=\frac{k_{\mathrm{B}}}{\alpha^{S} B}$.

\section{Discussion and Conclusions}

In this paper, we have derived the GENERIC formulation of the coarse-grained RaPiD model, and thus proved its thermodynamic admissibility and consistency. This verification essentially validates all previous results obtained with the RaPiD particles technique. Furthermore, the GENERIC framework allowed us to separate energetic contributions from entropic contributions to the dynamics of all ingredients in the model. In particular we have found an expression for the total stress, in which the distribution of entropic and energetic contributions is as follows: the rearrangements of the coronae's internal configurations together with their pair penetration forces are of entropic origin and contribute to all occurrences of the stress; the energetic interactions between the RaPiD particles contribute to the reversible part of the stress. The expression for the total osmotic pressure tensor agrees with the one used in all RaPiD simulations so far. Since we based our approach on local properties, the total osmotic pressure tensor obtained is a local property as well. It agrees with the one obtained a long time ago by Schofield and Henderson (1982), and used for example by Schindler (2010). A simple derivation for stresses in Couette flow was further given by Thakre et al. (2008).

We have derived the set of coupled transport equations for the RaPiD model using the systematic GENERIC formulation of nonequilibrium thermodynamics. Besides the equations of motion for the total density, total momentum, and solvent energy, we have obtained the Smoluchowski equation for the time evolution of the particles' positions and penetration numbers. The corresponding stochastic differential equations are indeed identical to the ones used in RaPiD simulations, and we therefore confirm the validity of these equations. In particular, the forces occurring in these equations include both energetic and entropic contributions, materialized by the occurrence of the free energy $\Phi(\mathbf{r}, \mathbf{R}, \mathbf{n})=\Phi^{E}(\mathbf{R})-T(\mathbf{r}) \Phi^{S}(\mathbf{R}, \mathbf{n})$.

However, the linear Eq. (48) results from an approximation of $\phi^{S}$ given by Eq. 23 that is valid only for small deviations $n_{i j}-n_{0}$. The entropic nature of the interaction generated by pair penetration, highlighted by our GENERIC formulation, indicates that a more appropriate expression of $\phi^{S}$, also valid for large deviations, could take the typical form

$$
\begin{aligned}
\phi^{S}\left(\mathbf{R}_{i j}, n_{i j}\right)= & \alpha^{S}\left(\mathbf{R}_{i j}\right) n_{0}^{2}\left(\mathbf{R}_{i j}\right) \\
& \times\left[\frac{n_{i j}}{n_{0}\left(\mathbf{R}_{i j}\right)} \ln \frac{n_{i j}}{n_{0}\left(\mathbf{R}_{i j}\right)}+1-\frac{n_{i j}}{n_{0}\left(\mathbf{R}_{i j}\right)}\right] .
\end{aligned}
$$

This relation would then result in the following nonlinear equation for the time evolution of the penetration parameter:

$\frac{d n_{i j}}{d t}=-\frac{n_{0}}{\tau} \ln \frac{n_{i j}}{n_{0}}+\sqrt{2 B} \theta_{i j}$.

The functional form of the entropy given by Eq. 49 allows only for positive values of $n_{i j}$. It then appears to be a natural choice if $n_{i j}$ can indeed be interpreted as a count of contact and/or entanglement points for overlapping polymers, for example. In that case, Eq. 23 is in fact particularly questionable. We thus strongly advise the use of the above equation to describe the evolution of the penetration parameters in future simulations.

Further refinements of the model could include the account of hydrodynamic interactions between RaPiD particles. The latter affects neither the expressions for the energy and the entropy nor the choice of variables (Öttinger and Grmela 1997; Wagner 2001) but imposes a modification of the friction operator $\mathbf{M}$.

The RaPiD model has been recently used to model pressure sensitive adhesives (Padding et al. 2011). In 
this system, interfacial phenomena play an important role, and the formalism presented here could be extended to include such effects by means of the MINT brackets (Moving Interface Normal Transfer) recently implemented by Öttinger et al. (2009).

Acknowledgements The authors thank Martin Kröger for insightful discussions. Support provided by the European Commission through the MODIFY (FP7-NMP-2008-SMALL-2, Code 228320) research project is greatly acknowledged.

\section{References}

Adams JM, Fielding SM, Olmsted PD (2011) Transient shear banding in entangled polymers: a study using the Rolie-Poly model. J Rheol 55:1007-1032

Bird RB, Armstrong RC, Hassager O (1987) Dynamics of polymeric liquids, 2nd edn. Wiley, New York

Boukany PE, Wang SQ (2009) Shear banding or not in entangled DNA solutions depending on the level of entanglement. J Rheol 53:73-83

Briels WJ (2009) Transient forces in flowing soft matter. Soft Matter 5:4401-4411

Briels WJ, Vlassopoulos D, Kang K, Dhont JKG (2011) Constitutive equations for the flow behavior of entangled polymeric systems: application to star polymers. J Chem Phys $134: 124901$

Cao J, Likhtman A (2012) Shear banding in molecular dynamics of polymer melts. Phys Rev Lett 108:028302

Cates ME, Evans MR (eds) (2000) Soft and fragile matter: nonequilibrium dynamics, metastability and flow. Institute of Physics Publishing, Bristol, UK

Dhont JKG, Briels WJ (2008) Gradient and vorticity banding. Rheol Acta 47:257-281

Ellero M, Español P, Flekkøy E (2003) Thermodynamically consistent fluid particle model for viscoelastic flows. Phys Rev E 68:041504

Fuchs M, Cates ME (2009) A mode coupling theory for Brownian particles in homogeneous steady shear flow. J Rheol 53:9571000

Götze W (1999) Recent tests of the mode-coupling theory for glassy dynamics. J Phys: Condens Matter 11:A1-A45

Götze W, Sjörgen L (1992) Relaxation processes in supercooled liquids. Rep Prog Phys 55:241-376

Grmela M, Öttinger HC (1997) Dynamics and thermodynamics of complex fluids. I. Development of a general formalism. Phys Rev E 56:6620-6632

Hütter M, Svendsen B (2012) Thermodynamic model formulation for viscoplastic solids as general equations for nonequilibrium reversible-irreversible coupling. Contin Mech Thermodyn 24:211-227

Ilg P, Öttinger HC (1999) Nonequilibrium relativistic thermodynamics in bulk viscous cosmology. Phys Rev D 61:023510

Ilg P, Mavrantzas V, Öttinger HC (2009) Multiscale modeling and coarse graining of polymer dynamics: simulations guided by statistical beyond-equilibrium thermodynamics. In: Gujrati PD, Leonov AI (eds) Modeling and simulation in polymers. Wiley-VCH, Weinheim, Germany, pp 343-383

Irving JH, Kirkwood JG (1950) The statistical mechanical theory of transport processes. IV. The equations of hydrodynamics. J Chem Phys 18:817-829
Kindt P, Briels WJ (2007) A single particle model to simulate the dynamics of entangled polymer melts. J Chem Phys 127:134901

Kröger M (2005) Models for polymeric and anisotropic liquids. Lecture notes in physics, vol 675. Springer, New York

Kröger M, Hütter M (2010) Automated symbolic calculations in nonequilibrium thermodynamics. Comput Phys Commun 181:2149-2157

Larson RG (1998) The structure and rheology of complex fluids. Oxford University Press, Oxford, UK

McLennan JA (1989) Introduction to nonequilibrium statistical mechanics. Prentice Hall, Englewood Cliffs, New Jersey

Mielke A (2011) Formulation of thermoelastic dissipative material behavior using GENERIC. Contin Mech Thermodyn 23:233-256

Müller-Plathe F (2002) Coarse-graining in polymer simulation: from the atomistic to the mesoscopic scale and back. ChemPhysChem 3:754-769

van den Noort A, den Otter WK, Briels WJ (2007) Coarse graining of slow variables in dynamic simulations of soft matter. Europhys Lett 80:28003

Öttinger HC (1998a) On the structural compatibility of a general formalism for nonequilibrium dynamics with special relativity. Physica, A 259:24-42

Öttinger HC (1998b) Relativistic and nonrelativistic description of fluids with anisotropic heat conduction. Physica, A 254:433-450

Öttinger HC (1999) Thermodynamically admissible equations for causal dissipative cosmology, galaxy formation, and transport processes in a gravitational collapse. Phys Rev D 60:103507

Öttinger HC (2001) Thermodynamic admissibility of the pompon model for branched polymers. Rheol Acta 40:317-321

Öttinger HC (2005) Beyond equilibrium thermodynamics. WileyInterscience, Hoboken, New Jersey

Öttinger HC (2011) The geometry and thermodynamics of dissipative quantum systems. Europhys Lett 94:10006

Öttinger HC, Grmela M (1997) Dynamics and thermodynamics of complex fluids. II. Illustrations of a general formalism. Phys Rev E 56:6633-6655

Öttinger HC, Bedeaux D, Venerus D (2009) Nonequilibrium thermodynamics of transport through moving interfaces with application to bubble growth and collapse. Phys Rev E 80:021606

Padding JT, Mohite LV, Auhl D, Briels WJ, Bailly C (2011) Mesoscale modeling of the rheology of pressure sensitive adhesives through inclusion of transient forces. Soft Matter 7:5036-5046

Pagonabarraga I, Frenkel D (2001) Dissipative particle dynamics for interacting systems. J Chem Phys 115:5015-5026

Schindler M (2010) A numerical test of stress correlations in fluctuating hydrodynamics. Chem Phys 375:327-336

Schofield P, Henderson JR (1982) Statistical mechanics of inhomogeneous fluids. Proc R Soc Lond, A 379:231-246

Sprakel J, Spruijt E, van der Gucht J, Padding JT, Briels WJ (2009) Failure-mode transition in transient polymer networks with particle-based simulations. Soft Matter 5:47484756

Thakre AK, den Otter WK, Padding JT, Briels WJ (2008) Spinodal decomposition of asymmetric binary fluids in a microCouette geometry simulated with molecular dynamics. J Chem Phys 129:074505

Wagner NJ (2001) The Smoluchowski equation for colloidal suspensions developed and analyzed through the GENERIC formalism. J Non-Newtonian Fluid Mech 96: 177-201 